\title{
DESARROLLO ENERGÉTICO Y POLÍTICAS
}

\author{
Cristián Hermansen Rebolledo
}




\section{CRISTIÁN HERMANSEN REBOLLEDO}

Ingeniero Civil Electricista de la Universidad de Chile. Profesor adjunto y director del Diplomado en Regulación de Electricidad del Departamento de Ingeniería Eléctrica de la Universidad de Chile. Presidente de la Comisión de Energía del Colegio de Ingenieros de Chile. Desde 1994 es director gerente de ACTIC Consultores, empresa de asesoría en el sector energético y, anteriormente, fue jefe del Área Eléctrica de la Comisión Nacional de Energía y jefe de Tarifas y Costos en Chilectra. 


\section{DESARROLLO ENERGÉTICO Y POLÍTICAS}

\section{INTRODUCCIÓN}

El país, en los últimos años, ha debido enfrentar variadas situaciones de abastecimiento de energía eléctrica, predominando sequías, estrechez energética, falta de combustibles (como es el caso del gas natural), altos precios de combustibles y de la electricidad, y una incertidumbre respecto al abastecimiento futuro de energía eléctrica, tanto en las etapas de producción como de transporte de electricidad, y de sus precios para consumo residencial, minero e industrial.

La matriz eléctrica chilena depende en gran medida de las importaciones de combustibles, ya que solamente la hidroelectricidad es una energía nacional, junto a una pequeña generación eólica y de biomasa y, a futuro, se incorporará la solar. Las nuevas unidades de generación eléctrica, incorporadas en los últimos años, utilizan combustibles como carbón y petróleo importados, aumentando nuestra dependencia energética y la huella de carbono, pero con la ventaja de estar ubicadas cerca de los centros de consumo, por lo cual requieren de líneas de transmisión eléctrica de menor longitud, con un ahorro de inversión y de pérdidas de electricidad en la transmisión de energía.

Esta situación energética actual debe ser analizada en función de la legislación eléctrica y de las políticas de energía del Estado de Chile, ya sean implícitas o explicitas, para evaluar la existencia, vigencia y alcance de estas políticas en un sector con procesos de largo plazo.

No ha existido en forma explícita una política de desarrollo de los recursos energéticos del país, excepto por los desarrollos impulsados, a mediados del siglo pasado, por la Corporación de Fomento de la Producción (CORFO) dentro de un esquema empresarial donde se abordaron proyectos de energía para incrementar la industrialización del país.

Tampoco ha existido una política de reservas estratégicas físicas de combustibles, que es una política definida en algunos países como, por ejemplo, Estados Unidos.

De acuerdo a los Balances Nacionales de Energía editados por el Ministerio de Energía, la energía consumida en el país en los últimos veinte años es de origen importado en el orden del $70 \%$. Las únicas energías primarias totalmente nacionales son la leña, del orden del $20 \%$ de la matriz, y la hidroelectricidad, del orden del $8 \%$ dependiendo del año hidrológico.

El análisis se centrará en el sector eléctrico ya que, como se explicará más adelante, el país no tiene, ni desarrollada ni en proyecto, una ley de hidrocarburos. Esta actividad se desarrolla libremente, cumpliendo normas de seguridad tal como las panaderías, que deben cumplir con las normas sanitarias.

Se indicará primeramente la situación del país al ponerse en marcha la legislación eléctrica, los supuestos de competencia considerados, los parámetros económicos relevantes, 
y la sociedad que es sujeto de la aplicación de la electricidad y su evolución hasta estos días, junto con la evolución del sector eléctrico.

Estos elementos permitirán una mejor comprensión de los caminos recorridos y futuros, de las posibilidades energéticas del país y de las políticas públicas.

\section{HIDROCARBUROS}

El sector petróleo no dispone de una ley que regule el sector hidrocarburos, solamente la Ley No 9.618 del 16 de junio de 1950, Ley Orgánica de la Empresa Nacional del Petróleo (ENAP) determina que el Estado tiene la propiedad de los yacimientos de petróleo, y que las funciones y derechos del Estado son ejercidos por ENAP.

Posteriormente, en el año 1978, se liberaliza el mercado, se permite la libre importación y se establece la libertad de precios para los hidrocarburos.

También el Decreto Ley (DL) No 1.089 del año 1975 establece los Contratos Especiales de Operación Petrolera, en los que el Estado, como dueño del recurso, contrata con un tercero la exploración y explotación, en condiciones comerciales definidas en cada contrato.

La Ley $N^{\circ}$ 18.888, publicada el 6 de enero de 1990, establece que ENAP puede desarrollar dentro o fuera del territorio nacional, directamente o asociado con terceros, la exploración y explotación de hidrocarburos. Esta ampliación del giro de ENAP, realizado a través de su filial Sipetrol, le ha permitido asegurar en forma parcial el abastecimiento de petróleo al país, pero depende en el futuro de las políticas comerciales de dicha empresa y no es una política del país.

La exploración y explotación de los hidrocarburos queda en manos de una empresa (en esa época y en la actualidad de propiedad del Estado), dependiendo de las políticas y solidez financiera de esta empresa la labor de desarrollar estos recursos energéticos.

En general, la prospección y explotación de hidrocarburos por ENAP se ha centrado en la Región de Magallanes, para abastecer de gas natural a dicha región y para la obtención de petróleo, cuya producción en la actualidad es del orden del 1\% del petróleo consumido en el país.

Tal como ocurre en el sector eléctrico, no existe una política clara de desarrollo de los hidrocarburos y, además, en este caso, queda supeditada a las decisiones de la administración de turno y de los recursos financieros disponibles de una empresa con una multiplicidad de funciones de mayor urgencia.

En el sector gas natural también la explotación y exploración de este recurso energético ha sido realizada por ENAP, confundiéndose los roles empresariales, regulatorios y de investigación de los recursos.

Se puede concluir que existe una política energética para la explotación y exploración de los hidrocarburos, como el petróleo y gas natural, pero esta política depende de la sanidad económica y de las prioridades comerciales de una empresa en particular, por el momento estatal. Los resultados de la obtención de hidrocarburos han sido muy bajos, incrementándose la dependencia energética en estos combustibles. 


\section{ENTORNO DEL PAÍS AL INICIO DE LA REGULACIÓN ELÉCTRICA}

Las reformas en el sector económico en los años 70, incluyendo las energéticas, se iniciaron con la dictación en diciembre de 1973 del Decreto con Fuerza de Ley (DFL) No 211, Normas para la Defensa de la Libre Competencia, sucesora del Título V: Normas para Fomentar la Libre Competencia Industrial y Comercial, de la Ley No 13.305 del 6 de abril de 1959, la cual da las bases para el posterior desarrollo económico y liberalización de los diferentes mercados.

El DFL No 211 se dictó porque, entre otros, "resulta necesario garantizar la libre competencia, previniendo la existencia del monopolio y de las prácticas monopólicas, y sancionando drásticamente su ejecución".

Así, se califica como delito a los que tiendan a impedir la libre competencia, ya sea eliminar, restringir o entorpecer esta.

Además, en sus considerandos indica que "las prácticas monopólicas son contrarias a una sana y efectiva competencia en el abastecimiento de los mercados ya que, mediante el control de la oferta o demanda, es posible fijar precios artificiales y lesivos al interés del consumidor".

Es la existencia de libre competencia la que permite la ocurrencia de numerosos actores, tanto en oferta como en demanda, para posibilitar la determinación de niveles de precios óptimos y optar entre los diferentes agentes.

La legislación eléctrica aplicada a partir de 1980, como efecto de la creación de la Comisión Nacional de Energía (CNE) por medio del DL No 2.224 de 1978, y oficializada por medio del DFL No 1 del Ministerio de Minería publicado el 13 de septiembre de 1982, considera esta base económica y legal y se refiere a aspectos técnicos del sector, y solamente determina un sistema de fijación de precios para clientes pequeños por no constituir este un mercado competitivo.

Por otra parte, el DFL No 1.122, Código de Aguas, publicado el 29 de octubre de 1981, indica que las aguas terrestres son bienes nacionales de uso público y se otorga a los particulares el derecho de aprovechamiento de ellas.

Indica que "el derecho de aprovechamiento sobre las aguas es de dominio de su titular, quien podrá usar, gozar y disponer de él en conformidad a la ley".

A partir de 1981 se registran a nombre de particulares, incluyendo a las empresas eléctricas existentes, derechos de agua no consuntivos destinados a la generación eléctrica.

Por otra parte, Chile, a inicios de la década de los 80 , era un país que estaba implementando nuevas políticas económicas de participación predominantemente privada, apertura de mercados de importación y de exportación, eficiencia económica y competitividad, con variadas complejidades en el proceso de transición y de reemplazo de actividades económicas tradicionales.

El PIB per cápita en el año 1980 era de US\$2.767 y de US\$ 3.142 en 1981, lo que da cuenta de un país con desarrollo económico bajo, un alto nivel de pobreza, desempleo de $10.4 \%$ en 1980 y de $11.3 \%$ en 1981, una inflación de $33.1 \%$ en 1980 y un consumo eléctrico por habitante de $1.058 \mathrm{KWH}$ por año. 
En la actualidad, Chile ha alcanzado, de acuerdo a cifras del Banco Mundial, un PIB per cápita de US $\$ 16.000$, esperando llegar a US\$20.000 en los próximos años, y un consumo por habitante al año 2009 de $3.300 \mathrm{KWH}$ por habitante. Este consumo, comparado con zonas como Europa y California de valores sobre $6.000 \mathrm{KWH}$ por habitante, indica que el crecimiento eléctrico se debe incrementar acorde con el mejor nivel económico de la población, superando el efecto de un uso eficiente de la energía.

La ciudadanía y, en este caso específico, los consumidores, no tenían presencia ni representación, ya que al ser las empresas eléctricas mayoritariamente estatales, las consideraban parte del Estado ante el cual no podían influir. Tampoco los afectados se oponían a la constitución de servidumbres de paso, o de ocupación de terrenos para líneas de trasmisión, o de centrales hidráulicas por considerar, en muchos casos, en forma anticipada, que se resolvería a favor de la empresa eléctrica.

Tampoco existía en esa época la comprensión de temas como el calentamiento global, gases de efecto invernadero, huella de carbono, que son los elementos que preocupan a la sociedad planetaria del siglo XXI.

La labor de ENDESA estatal, desde su creación hasta la década de los 80, fue realizar en la práctica la labor de un instituto de electrificación del país, detectando los recursos de agua aprovechables para la generación hidroeléctrica y construyendo centrales de generación, anticipándose a los crecimientos de la demanda eléctrica a lo largo de todo el país.

La labor de esta empresa, unida a la visión del abastecimiento eléctrico como una función de estrategia y de seguridad nacional, no en forma explícita pero sí en la práctica, condicionan una nueva legislación que está dirigida a permitir la competencia entre los actores del sector eléctrico, pero no a preocuparse ni a incentivar el desarrollo de los recursos energéticos en forma explícita.

La legislación eléctrica establece una separación de funciones en el sector eléctrico para superar la confusión de los roles empresariales, regulatorios y de investigación de recursos energéticos desarrollados por ENDESA, lo cual le confería una posición dominante en el mercado, pero no contempla la definición de una política de desarrollo de los recursos energéticos del país.

En este sentido, las empresas industriales y mineras emprendían sus nuevos proyectos con la seguridad de contar con suministro eléctrico a un precio razonable, situación que con la nueva legislación debía mantenerse al existir competencia en generación.

Adicionalmente, en el sector energía, con un país gobernado por las Fuerzas Armadas, se producía la situación de tener como presidente de la CNE a un ministro teniente general que, por la verticalidad de mando de la estructura militar, tenía mayor autoridad que los generales o almirantes que dirigían las empresas eléctricas, lo cual llevó en ese período a una interpretación única de la legislación de acuerdo a los dictados de la CNE.

La legislación eléctrica desarrollada por la Comisión Nacional de Energía refleja procesos y algoritmos para cálculo de tarifas de generación, transmisión y distribución de electricidad, con el objeto de permitir la separación de estas actividades y la creación de empresas regionales de distribución eléctrica a partir de las respectivas áreas de Chilectra y de ENDESA. 
La legislación se supone neutra con respecto a los distintos recursos energéticos del país, sin embargo, sus elementos de tarificación y coordinación reflejan un enfoque en un sistema resultante de una combinación de recursos hidráulicos y térmicos.

También, en la nueva legislación, se define la obligatoriedad de dar servicio a todos los clientes en el área de concesión de las empresas distribuidoras, y se define la existencia de "clientes a precio libre" para aquellos con una potencia conectada superior a $2.000 \mathrm{KW}$, ya que supone la posibilidad de poder negociar su contrato de suministro eléctrico y de obtener mejores condiciones que los clientes regulados.

Este mercado de clientes libres operando en condiciones de competencia solamente comenzó a desarrollarse a mediados de la década de los 90, producto de la llegada del gas natural argentino a nuestro país.

\section{LEGISLACIÓN ELÉCTRICA EN LA DÉCADA DE LOS 80}

La nueva política eléctrica comenzó en forma práctica con la determinación de las tarifas a clientes finales, aplicada a partir del año 1980, y se ratifica con la publicación, el 13 de septiembre de 1982, del DFL No 1 del Ministerio de Minería la Ley General de Servicios Eléctricos.

La política energética no se explicita en la legislación, solamente al término de ese período de gobierno la CNE emite el libro El sector energía en Chile, en diciembre de 1989, donde define la estrategia energética.

En este documento se indica que "la estrategia que orienta el desarrollo del sector energía en Chile es consistente con la política general de desarrollo económico y social del país", a través de eficiencia económica en el sector energía.

También, indica el objetivo de "tener una independencia energética razonable para el país, promoviendo el desarrollo de todos los recursos nacionales que resulten económicamente convenientes en relación con los energéticos importados, y considerando una adecuada diversificación de las fuentes de suministro para estos últimos".

Solo en este párrafo existe una referencia al desarrollo de los recursos energéticos del país, pero este objetivo no se refleja en la redacción de la legislación eléctrica y queda supeditado a su competitividad con energéticos importados.

Este principio se aplicará en la década de los 90 con la incorporación a la matriz eléctrica chilena del gas natural argentino que, por su bajo costo, frenó el desarrollo de proyectos hidroeléctricos en el país.

En este mismo documento se expresan los objetivos de la nueva ley eléctrica de "establecer un conjunto de reglas del juego lo más claras y objetivas posible" para poder tener "un desarrollo eficiente y estable del sector e incentivar la participación del capital privado".

Agregando así elementos para reducir la burocracia del sector y tener los instrumentos de control para un buen funcionamiento del sector eléctrico.

Asimismo, en la explicitación, en dicho documento, de los criterios generales utilizados en la confección de la legislación eléctrica se indica: "El criterio básico de la ley 
eléctrica es propender el establecimiento de condiciones de competencia en este sector, reservando la acción reguladora del Estado solo para aquellas actividades que revisten características de monopolio natural".

También, al explicitar los criterios generales de la ley, el documento determina el funcionamiento libre en generación y transmisión, y regulado en distribución eléctrica.

La labor de desarrollo de nuevas centrales de generación eléctrica, por parte de las empresas estatales ENDESA y Chilectra, para el abastecimiento público, y de Codelco para abastecer sus consumos mineros, permite adelantarse a los crecimientos de la demanda y dar un abastecimiento eléctrico seguro, excepto en casos de sequías extremas como fueron los años 1988 y 1989.

Las empresas generadoras, en especial ENDESA, tenían una amplia cartera de proyectos con distintos grados de profundización de sus especificaciones para el abastecimiento eléctrico del país, con un enfoque a priorizar la componente hidroeléctrica para no depender de un combustible fósil como el carbón, en general, de procedencia importado por sus menores costos y mejor calidad.

La legislación permitió una optimización de los recursos de producción y de costos al definir un centro de despacho económico de generación, con una operación independiente de los contratos de suministro de las empresas de generación eléctrica.

Además, en esta época, al ser las empresas generadoras de propiedad estatal, sus inversiones debían ser aprobadas por la CNE y se adelantaban en forma eficiente a las necesidades de crecimiento del país, pero no enfocadas al uso de los recursos del país, sino a minimizar los costos de inversión, operación, mantenimiento y falla de suministro.

El supuesto implícito de la legislación es la existencia de una amplia oferta de nuevos proyectos en estudio y en construcción, así como el ingreso de nuevas empresas eléctricas para aumentar la competencia en el sector eléctrico.

Por lo tanto, no existen referencias explícitas para utilizar las posibilidades energéticas del país ni una política de apoyo a la investigación o explotación de los recursos.

La legislación fue creada centrándose en producir competencia entre las dos principales empresas generadoras existentes a la época, no contemplando el posible caso de, por ejemplo, incorporar minicentrales hidroeléctricas al sistema, debiendo estas cumplir con las mismas exigencias y condiciones de centrales de gran escala, requisitos que son fáciles de cumplir para empresas medianas y de gran tamaño, pero onerosas para pequeñas unidades independientes.

No se considera la participación ciudadana en la regulación, siendo la sociedad objeto pasivo de las fijaciones tarifarias, con escaso acceso a información pública relevante y con una centralización de las decisiones de regulación consideradas implícitamente en Santiago, lugar de domicilio legal de las gerencias de empresas eléctricas y autoridades del gobierno de turno.

Los estudios de recursos hidroeléctricos realizados en su época por ENDESA, y actualizados por especialistas, indican una potencia instalable en proyectos de mediano y gran tamaño superior a $11.000 \mathrm{MW}$, tanto en la zona central, del orden de los 5.200 MW, y de Aysén, del orden de los 5.800 MW. 
Por otra parte, un estudio de la Comisión Nacional de Riego en conjunto con la CNE, de octubre de 2007, indica un aprovechamiento hidroeléctrico en proyectos concretos utilizando canales de regadío del orden de los 860 MW en centrales superiores a 2 MW de capacidad.

\section{SITUACIÓN EN LA DÉCADA DE LOS 90}

A comienzos de la década de los 90, la CNE, en su función de preparar los planes y políticas del Plan Nacional de Desarrollo, se dedica a incentivar y adecuar la legislación para la introducción de un nuevo combustible: el gas natural argentino. Por lo demás, esta es la única función adicional a sus funciones principales de análisis de normas técnicas, de estructuras, y niveles de precios en el sector de energía.

Lo anterior, con el objetivo de posibilitar la apertura de mercados, diversificar la matriz energética, atraer nuevos operadores al sector eléctrico y permitir una mayor competencia en un sector con una fuerte concentración de la propiedad de los derechos de agua.

La preocupación estaba en desarrollar competencia, ya que no está definida una política de utilización de los recursos energéticos del país, y también es en este período que se produce el cierre de las principales minas de carbón al no poder competir con el carbón importado.

Estas políticas son correctas de acuerdo a las definiciones legales de promover la competencia y la eficiencia, que son los fundamentos implícitos en el DFL $N^{\circ} 1$.

El país, implícitamente, tiene la política de no tener reservas físicas de combustibles de cualquier tipo, y tampoco se consideran, desde un punto de vista estratégico, las reservas de agua en los embalses, sino solamente por su valor comercial futuro.

Al disponer de gas natural barato y abundante para la generación eléctrica, con facilidad de ubicación de sitios, cortos tiempos de construcción y bajos costos para instalar centrales de ciclo combinado, se frena el desarrollo de centrales hidroeléctricas medianas y pequeñas.

En un comienzo, las empresas generadoras existentes compiten por entrar a este nuevo mercado de generación con gas natural y se produce una sobreoferta de energía por un período de tiempo.

Esta disponibilidad de energía económica, que produce una baja de precios pagados por los clientes libres, permite que se inicie la operación del mercado de clientes libres y la competencia de las empresas generadoras por abastecer a estos clientes.

Durante esta década, se discute la Ley de Concesiones de Exploración (para determinar la potencialidad del recurso), y de Explotación (para la extracción y producción) de energía geotérmica, con el objeto de incorporar esta nueva fuente de energía y permitir el desarrollo de este recurso.

Es, en la práctica, el esbozo de la primera política pública enfocada en un recurso energético, y regula las relaciones entre las partes: concesionarios de energía geotérmica, Estado, dueños de terrenos, pertenencias mineras, contratos de operación petroleros, explotación y exploración de hidrocarburos, y derechos de aprovechamiento de aguas. 
Sin embargo, el resultado práctico, a la fecha, es no tener una central de energía geotérmica operando, a pesar de existir varias empresas trabajando en el tema.

\section{SITUACIÓN EN EL SIGLO XXI}

En esta época, se producen los primeros cambios importantes a la legislación eléctrica para solucionar los problemas de un mercado que no opera en forma competitiva.

La Ley $N^{\circ}$ 19.940, denominada Ley Corta I, en el año 2004 introduce modificaciones al DFL $N^{\circ} 1$, principalmente, para solucionar los problemas regulatorios y de competencia en el transporte de electricidad.

Un elemento incluido en estas modificaciones legales es la excepción de pago de peaje por el uso de las instalaciones de transmisión troncal para las energías renovables hasta una capacidad inferior a 9 MW, y de un descuento parcial para las centrales de generación eléctrica renovable inferior a 20 MW, situación que se mantiene hasta que la capacidad conjunta de estas centrales y sus peajes exentos de pago constituyan el 5\% de la capacidad del sistema, a partir del cual comenzarán a pagar sobre el diferencial que supere este $5 \%$.

Sin embargo, esta excepción del pago de peajes solamente se aplica a la transmisión troncal, debiendo las energías renovables pagar los peajes por los sistemas de transmisión adicional, subtransmisión y distribución.

En el año 2005, la Ley No 20.018, denominada Ley Corta II, introduce el concepto de licitaciones de suministro para abastecer el consumo de los clientes regulados de las empresas distribuidoras, pero su normativa favorece a las empresas existentes y dificulta la incorporación de nuevas empresas al sector. Esto se refiere a plazos cortos para iniciar el suministro, inferiores al plazo de construcción de las centrales y que, por lo tanto, no permiten el desarrollo de proyectos que utilicen los recursos energéticos del país.

La Ley No 20.257, en el año 2008, entrega una señal de diversificación de los recursos energéticos hacia las energías renovables no convencionales, al determinar que el $10 \%$ de la energía consumida debe provenir de fuentes renovables como son la energía hidráulica, limitada a una capacidad inferior a 20 MW, biomasa, geotérmica, solar, eólica y energía de los mares.

La crisis de las restricciones de suministro y posterior corte, prácticamente total, de la llegada de gas natural argentino, produce una reacción del gobierno de la época, presionándolo a generar una solución a corto plazo para evitar cortes de electricidad y mantener una seguridad energética, impulsando así la construcción de terminales de recepción y regasificación de gas natural licuado en Quintero y Mejillones.

Con un largo período de análisis, en la década de los 90, del recurso de energía geotérmica, el 7 de enero de 2000 se publica la Ley No 19.657 sobre Concesiones de Energía Geotérmica, pero su reglamento, a través del Decreto $N^{\circ} 32$, se publica en el año 2004 , siendo derogado y modificado por el Decreto $N^{\circ} 114$, publicado el 8 de marzo de 2013. 
El resultado práctico, a la fecha, es no tener en operación ninguna central que genere electricidad aprovechando el recurso de energía geotérmica.

Otro tema de análisis y discusión ha sido la generación residencial, por medios renovables no convencionales, de usuarios finales regulados con capacidad instalada inferior a $100 \mathrm{KW}$, que a semejanza de otros países, pudiesen intercambiar energía con el sistema de distribución. Sin embargo, la Ley No 20.571, publicada el 22 de marzo de 2012, no establece la igualdad de precios y define que un reglamento determinará las condiciones de operación, reglamento que todavía no ha sido emitido, habiendo transcurrido un año y medio a la fecha.

En el año 2008, la CNE publica el documento Política energética: Nuevos lineamientos, indicando que los desarrollos energéticos son procesos de largo plazo, y que se requiere "una política estable coherente con la política global de desarrollo nacional". Se plantea una estrategia de largo plazo para tener energía eléctrica sustentable, suficiente y segura, pero no se analiza en profundidad el desarrollo de los recursos energéticos del país.

La Ley No 20.402, publicada el 3 de diciembre de 2012, crea el Ministerio de Energía para agrupar todas las funciones y organismos del área energía distribuidos en diversas instituciones, manteniendo en lo esencial las funciones tradicionales y sin un mandato explícito de desarrollar los recursos energéticos del país.

En el año 2011, se desarrolla un proceso de discusión y análisis de las condiciones vigentes y futuras del sector eléctrico, por medio de dos procesos en paralelo: uno, la Comisión Asesora para el Desarrollo Eléctrico (CADE), convocada por el gobierno; y, por otra parte, la Comisión Ciudadana Técnico Parlamentaria (CCTP).

Ambos organismos analizan y entregan propuestas de los temas de competencia, sustentabilidad, eficiencia energética, y no tienen el foco principal en el uso de recursos energéticos del país, excepto la CCTP, que prioriza las energías renovables no convencionales, pero deja afuera la generación con recursos hidroeléctricos superiores a 20 MW de capacidad instalada.

Al inicio del año 2012, el Presidente de la República publica la Estrategia Nacional de Energía 2012-2030, que indica la necesidad del país de tener una estrategia de Estado de largo plazo situando el tema energético por sobre los gobiernos de turno.

Uno de los elementos de esta estrategia es desarrollar las energías renovables no convencionales, así como también las energías tradicionales, incluyendo la hidroelectricidad.

Sin embargo, en el año y medio transcurrido desde el lanzamiento de esta estrategia de energía, no se han concretado aspectos que posibiliten el desarrollo de los recursos energéticos del país, permaneciendo como una declaración de buenas intenciones.

\section{SITUACIÓN ACTUAL}

En la actualidad, la legislación no considera el desarrollo energético del país, ya que existe una estrategia a corto plazo de las empresas y de la autoridad para abordar el suministro eléctrico. 
La política a implementar no debiese centrarse ni tener como objetivo el otorgamiento de subsidios a algún tipo de tecnología -ya que estos distorsionan el mercado-, sino generar una política pública con las condiciones de mercado para posibilitar el desarrollo de los recursos del país. Esta política debiese ampliar la composición de los recursos energéticos y no obligarlos a encuadrarse en los requerimientos de las centrales térmicas.

La legislación, de alguna manera, está enfocada en defender a las empresas existentes en vez de desarrollar explícitamente una economía de mercado que favorezca el ingreso de nuevas empresas y el desarrollo de los recursos energéticos propios del país. En especial, potenciar el desarrollo de los recursos hidráulicos, geotérmicos, solares, biomasa, eólicos y, a futuro, energía de los mares, ya sea de mareas o de olas, recordando que hace dos décadas el recurso hidroeléctrico constituía el 65\% de la matriz eléctrica, y en la actualidad bordea el 30\%.

No existen, en la legislación actual, los mecanismos que contemplen las características especiales de estos recursos, sino que deben adaptarse a competir con los parámetros y características de las centrales térmicas a carbón, a gas natural o diesel. Tampoco existe este desarrollo para las centrales nucleares.

Los procesos de energía son de largo plazo, por lo que las políticas definidas en un gobierno se aplicarán en los gobiernos siguientes; por lo tanto, los gobiernos de turno se han inclinado más por soluciones de corto plazo, como la instalación de generación diesel, que es una solución rápida para evitar los cortes de suministro, pero que no ayuda al aprovechamiento de los recursos energéticos del país, ni a mediano ni a largo plazo.

Adicionalmente, estas soluciones de corto plazo, y de postergación de la utilización de los recursos energéticos como, por ejemplo, hidroelectricidad y geotermia para la generación eléctrica, han conducido a un elevado valor de los precios de la electricidad, con lo cual el país está perdiendo competitividad.

Es de esperar que las condiciones de abastecimiento eléctrico, incierto a partir del año 2015, permitan generar en el corto plazo una respuesta país para concretar soluciones que solamente rendirán resultados a partir del 2018, utilizando los recursos energéticos nacionales y disminuyendo nuestra dependencia energética y nuestra contribución al calentamiento global. 